\title{
Breast Cancer Treatment in a Patient with Decubitus Ulcer Infection Secondary to Spina Bifida: Surgical Resection versus Neoadjuvant Chemotherapy
}

\author{
Fumiya Sato ${ }^{a, b} \quad$ Akihiko Shimomura $^{a}$ Kanako Nakayama $^{b}$ \\ Yukino Kawamura ${ }^{a}$ Kazuki Hashimoto ${ }^{b}$ Yuko Ishibashi ${ }^{b}$ \\ Chikako Shimizu $^{a}$ Dai Kitagawa ${ }^{b}$ \\ ${ }^{a}$ Department of Breast and Medical Oncology, National Center for Global Health and \\ Medicine, Tokyo, Japan; breast Surgery Division, Department of Surgery, National Center \\ for Global Health and Medicine, Tokyo, Japan
}

\author{
Keywords \\ Breast cancer $\cdot$ Chemotherapy · Decubitus ulcer infection $\cdot$ Spina bifida
}

\begin{abstract}
Spina bifida (SB) is a congenital neural tube defect that often presents with neurological disability and decubitus ulcers. A 66-year-old woman with SB presented to our hospital with decubitus ulcers and was treated by a plastic surgeon. She was referred to our department because of a mass measuring $5 \times 4 \mathrm{~cm}$ in the superolateral quadrant of the right breast. The size of the right axillary lymph node $(\mathrm{LN})$ was $2 \times 1 \mathrm{~cm}$. A core-needle biopsy revealed an invasive ductal carcinoma. Total mastectomy and axillary LN dissection were planned. However, 2 days prior to surgery, the size of the mass and the LN rapidly increased to $7 \times 4 \mathrm{~cm}$ and $3 \times 2 \mathrm{~cm}$, respectively. Furthermore, the enlarged LN was close to the thoracodorsal artery. Since complete resection was difficult, neoadjuvant chemotherapy was also administered. On day 11 of neoadjuvant chemotherapy, the patient was febrile and developed a decubitus ulcer infection at the buttock. The neutrophil count was within normal limits; thus, she was not diagnosed with febrile neutropenia. Follow-up computed tomography revealed a shrinking of the mass to $5 \times 4 \mathrm{~cm}$ after the first cycle of neoadjuvant chemotherapy. After 17 days of antibiotic therapy and drainage, total mastectomy and axillary LN dissection were performed. Due to the risk of recurrence of infection, adjuvant chemotherapy was discontinued and hormone therapy was initiated. In conclusion, indications for chemotherapy should be carefully evaluated in SB patients with lower limb paralysis and decubitus ulcers.
\end{abstract}




\section{Introduction}

The survival rate and life expectancy of patients with spina bifida (SB) has greatly increased with the advent of modern therapies. However, due to this prolonged life expectancy, SB patients are exposed to the risk of adult-onset malignancies. Here, we present the case of a 66-year-old female with SB and breast cancer. We planned to manage the patient via complete surgical resection. However, a rapid increase in breast tumor size was observed; thus, neoadjuvant chemotherapy was administered. During this treatment period, the patient developed decubitus ulcer infection. Therefore, neoadjuvant chemotherapy was discontinued, and we proceeded with the operation.

\section{Case Presentation}

A 66-year-old woman with SB (Fig. 1) presented to our hospital with decubitus ulcers due to lower limb paralysis secondary to SB and was treated accordingly by a plastic surgeon. She had a prior allergic event due to a contrast agent. She was referred to our department because of a mass in her right breast. This mass measuring $5 \times 4 \mathrm{~cm}$ in the superolateral quadrant of the right breast was first detected on palpation. Mammography revealed that the mass was categorized as category 4 (Fig. 2a). Breast ultrasound also revealed a mass (Fig. 2b, above) and an enlarged right axillary lymph node (LN) measuring $2 \times 1 \mathrm{~cm}$ (Fig. $2 \mathrm{~b}$, below). Computed tomography (CT) also showed a mass (Fig. 2c, above) and an enlarged right axillary LN measuring $2.5 \times 2 \mathrm{~cm}$ (Fig. 2c, below), with no observed invasion or metastasis. Based on a core-needle biopsy, the mass was diagnosed as an invasive ductal carcinoma (IDC): estrogen receptor (ER)-positive, progesterone receptor (PgR)-positive, human epidermal growth factor receptor type2 (HER2)-negative, Ki-67 20\%, cT3N1M0, and cStage IIIA. Total mastectomy and axillary LN dissection were planned. However, 2 days prior to surgery, the size of the mass and LN rapidly increased. Breast ultrasound showed a mass measuring $7 \times 4$ cm (Fig. 3a) and an enlarged LN measuring $3 \times 2 \mathrm{~cm}$ (Fig. 3b, above). The enlarged LN was poorly mobile and compressed the thoracodorsal artery due to its proximity (Fig. 3b, below). Therefore, we decided that complete surgical resection would not be appropriate, and neoadjuvant chemotherapy with doxorubicin $60 \mathrm{mg} / \mathrm{m}^{2}$ and cyclophosphamide $600 \mathrm{mg} / \mathrm{m}^{2}$ (AC) therapy was administered. Pegylated filgrastim $(3.6 \mathrm{mg})$ was administered a day after the first AC. On day 11 of the first cycle of AC therapy, the patient was febrile and developed a decubitus ulcer infection at the buttock. Blood tests revealed an inflammatory response (white blood cell count, 13,640/ $\mu \mathrm{L}$; C-reactive protein level, $14.7 \mathrm{mg} / \mathrm{dL}$ ). Since her neutrophil

Fig. 1. A protrusion of the meninges (arrow) through a defect point in the spine on a CT scan in a prone position.

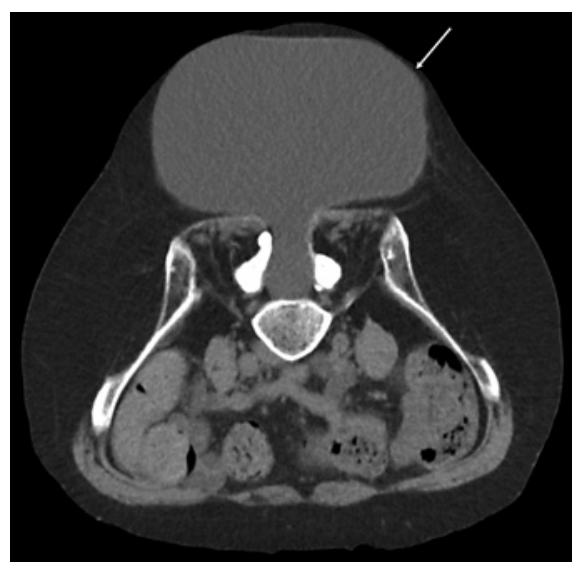




\section{Case Reports in Oncology}

Sato et al.: Breast Cancer Treatment in a Patient with Spina Bifida

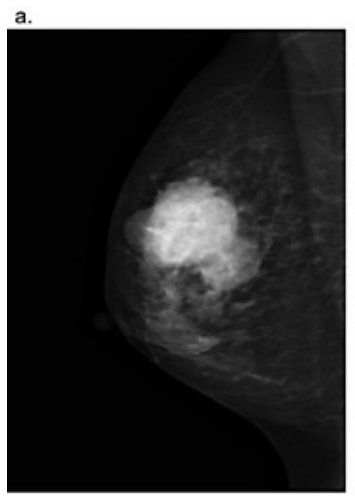

c.
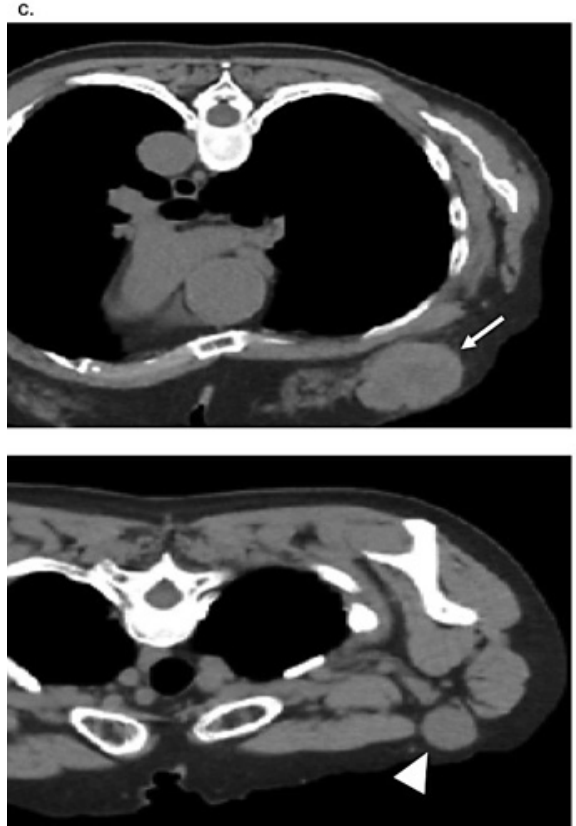
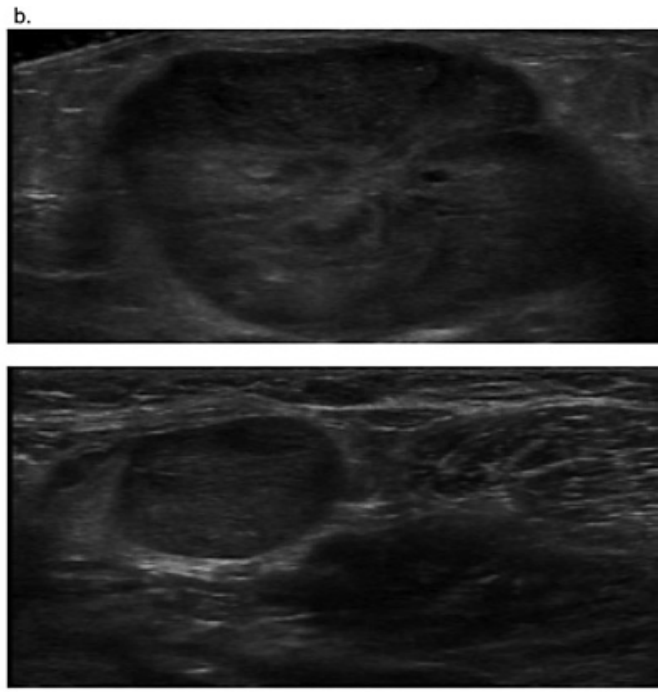

Fig. 2. MG, US, and CT imaging of the right breast and axilla. a MLO (left) and CC (right) views of the right breast on a mammography, showing a $5 \times 4$-cm mass in the upper outer quadrant, category 4 . b US imaging of the right breast (above) and axilla (below), showing a $5 \times 4-\mathrm{cm}$ mass in the superolateral quadrant (above) and enlarged right axillary LN, $2 \times 1 \mathrm{~cm}$ (below). c Axial view of the breast (above) and axilla (below) on a CT scan in a prone position, showing a $5 \times$ 4-cm mass in the right breast (arrow) and an enlarged $2.5 \times 2-\mathrm{cm} \mathrm{LN}$ at the right axilla (arrowhead). CC, craniocaudal; CT, computed tomography; LN, lymph node; MG, mammography; MLO, mediolateral oblique; US, ultrasound.

count was within normal limits, we ruled out a febrile neutropenia (FN). Even though granulocyte colony-stimulating factor was administered, the AC was deemed intolerable for this patient. Antibiotic therapy and drainage for decubitus ulcer infection were required. Followup CT after one cycle of AC showed that the size of the mass had reduced from $7 \times 4 \mathrm{~cm}$ to 5 $\times 4 \mathrm{~cm}$ and that the LN did not significantly compress the thoracodorsal artery. Therefore, we decided to proceed with complete surgical resection. After 15 days of antibiotic therapy and drainage, her inflammatory markers improved (white blood cell count, 7,450/ $\mu \mathrm{L}$; C-reactive protein level, $1.07 \mathrm{mg} / \mathrm{dL}$ ). After 17 days of antibiotic therapy and drainage, total mastectomy and axillary LN dissection were performed. A circular cushion enabled her to maintain a lateral position during the surgery to avoid pressure on the meningocele. The operating time was $2 \mathrm{~h}$ and $3 \mathrm{~min}$, and the total blood loss was $38 \mathrm{~mL}$.

Her postoperative course was uneventful. The pathological findings were IDC, ER-positive, PgR-positive, HER2-negative, Ki-67 2\%, and pT3N1aM0. The patient was discharged on postoperative day 12 with oral antibiotic therapy for decubitus ulcer infection. Due to the risk of recurrence of infection, adjuvant chemotherapy was discontinued, and hormone therapy was initiated. No cancer recurrence was observed 10 months after the operation. 


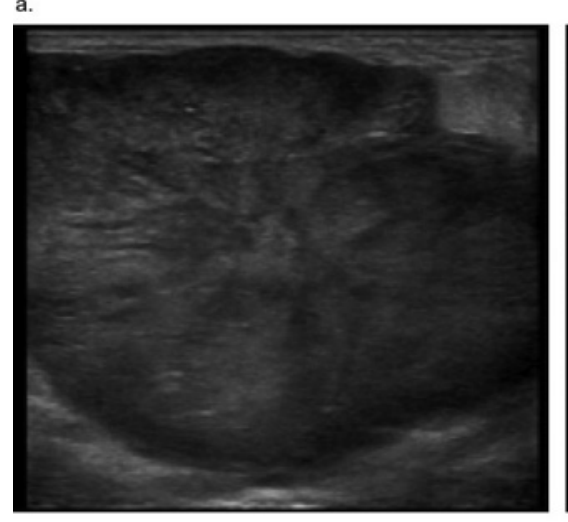

Fig. 3. US imaging of the right breast and axilla. a Enlarged mass in the right breast, from $5 \times 4 \mathrm{~cm}$ to $7 \times 4 \mathrm{~cm}$. b Enlarged LN (arrowhead) at the right axilla in close proximity to the thoracodorsal artery (arrow). LN, lymph node; US, ultrasound.

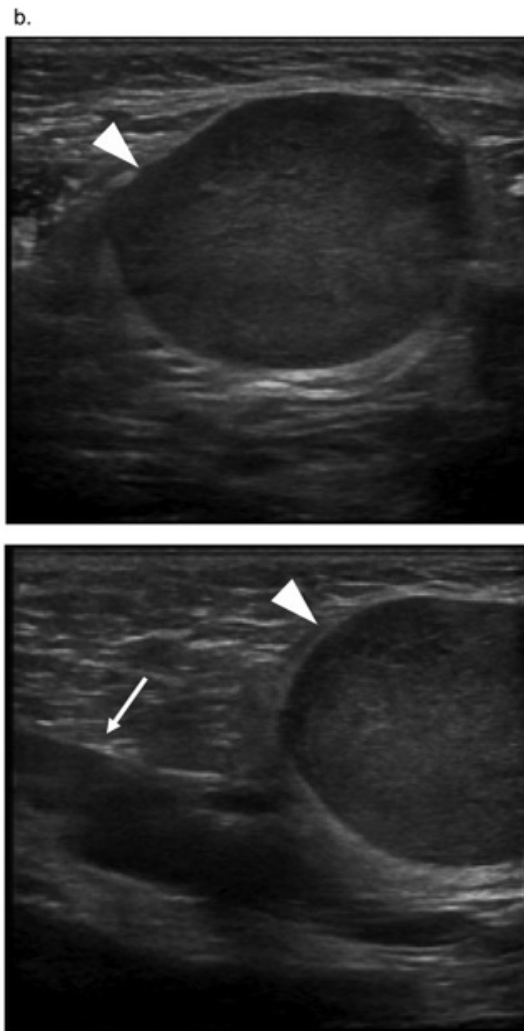

\section{Discussion}

Breast cancer in patients with SB is extremely rare. Due to medical advancements, nearly all children born with SB survive into adulthood, although most have a neuropathic bladder with potential complications of incontinence, infection, renal injury, and diminished quality of life [1]. Owing to this increase in life expectancy, patients are at a greater risk of developing adult-onset malignancies. However, breast cancer is rare as an adult-onset malignancy in patients with SB. A search for published articles on breast cancer with SB was conducted in the PubMed database on December 2, 2020. The search words were ("bifida" OR "meningocele" OR "myelomeningocele") AND ("cancer" OR "malignancy" OR "tumor") AND ("breast" OR "mammo") in the title and/or abstract. Five articles were identified. Among these, there were 2 cases of breast cancer with SB [2,3], and the present report is the third. The characteristics of these 3 cases are summarized in Table 1 . The cases included 2 middle-aged women. One was treated with surgery and adjuvant chemotherapy (epirubicin $100 \mathrm{mg} / \mathrm{m}^{2}$ with 5 -fluorouracil $500 \mathrm{mg} / \mathrm{m}^{2}$ and cyclophosphamide $500 \mathrm{mg} / \mathrm{m}^{2}$ [FEC100]), and the other's treatment was not described. One developed urinary and fecal disorders because of FEC100. In our case, surgical resection was performed after one cycle of AC.

In the present case, the hormone receptor-positive tumor in the right breast increased rapidly in only 2 months, making it inoperable; preoperative therapy was then considered. According to Diéras et al. [4] and Bear et al. [5], the occurrence of infection and FN in AC were lower than those in regimens including taxanes. However, the patient developed fever and infection during the first cycle of AC. Her neutrophil count was within normal limits; therefore, an infection was considered. Before the tumor was detected, she was given routine management for long-term decubitus ulcers due to lower limb paralysis secondary to SB.

\section{Karger'}


Table 1. Summary of breast cancer with SB

\begin{tabular}{|c|c|c|c|c|c|c|c|c|c|c|}
\hline & Reference & Year Age/sex & Site & $\begin{array}{l}\text { Size, } \\
\mathrm{cm}\end{array}$ & TNM & Stage & Surgery & $\begin{array}{l}\text { Chemo- } \\
\text { therapy }\end{array}$ & Complication & Outcome \\
\hline Case 1 & Hoy et al. [2] & 2013 66/F & Left breast & $\mathrm{N} / \mathrm{D}$ & T2N0M0 & Stage IIA & $\mathrm{Bt}+\mathrm{Ax}$ & FEC100 & $\begin{array}{l}\text { Fecal and urinary } \\
\text { disorder }\end{array}$ & Alive \\
\hline Case 2 & $\begin{array}{l}\text { Malla et al. } \\
\text { [3] }\end{array}$ & 2016 50/F & Left breast & $\mathrm{N} / \mathrm{D}$ & $\mathrm{N} / \mathrm{D}$ & $\mathrm{N} / \mathrm{D}$ & $\mathrm{N} / \mathrm{D}$ & $\mathrm{N} / \mathrm{D}$ & $\mathrm{N} / \mathrm{D}$ & Dead \\
\hline Case 3 & Our case & 2020 66/F & $\begin{array}{l}\text { Right } \\
\text { breast }\end{array}$ & 5 & T3N1M0 & Stage IIIA & $B t+A x$ & $\mathrm{AC}$ & Infection & Alive \\
\hline
\end{tabular}

AC, doxorubicin and cyclophosphamide; Ax, axillary lymph node dissection; Bt, total mastectomy; FEC100, epirubicin $100 \mathrm{mg} / \mathrm{m}^{2}$ with 5-fluorouracil $500 \mathrm{mg} / \mathrm{m}^{2}$ and cyclophosphamide $500 \mathrm{mg} / \mathrm{m}^{2}$; N/D, no data.

Considering her background, she should have been considered as a high-risk patient for infection even if the neoadjuvant chemotherapy was AC. Ideally, surgical resection should have been performed before the tumor and LN are increased in size.

According to Horner-Johnson et al. [6], women with disabilities (especially complex or severe) are less likely to be up-to-date with breast cancer screening. In this study, the disability severity variable was coded as 1 (no limitations; none), 2 (basic action difficulties only), 3 (complex activity limitations only), and 4 (basic action difficulties and complex activity limitations; both). She was coded as 4 for the disability severity variable [7]. She had never undergone previous breast cancer screening. Had her breast mass been detected earlier, it could have been easily and completely resected without a rapid increase in size or any infection.

\section{Conclusion}

Breast cancer is a rare adult-onset malignancy in patients with SB. Early intervention is primordial because these patients are at a high risk of complications, including infection. However, early detection of breast cancer is less likely in women with severe disability; therefore, it is important to raise awareness of breast cancer screening in women with disabilities at the appropriate age. For advanced-stage breast cancer, indications for chemotherapy should be evaluated carefully, especially in patients with severe disability such as SB patients with decubitus ulcers due to lower limb paralysis.

\section{Statement of Ethics}

Written informed consent for publication of this case report and any accompanying images was obtained from the patient.

\section{Conflict of Interest Statement}

A.S. received grants from AstraZeneca, grants from Chugai Pharmaceutical, grants from Daiichi-Sankyo, grants from Taiho Pharmaceutical, grants from Mochida Pharmaceutical, outside the submitted work. C.S. received grants from Eli Lilly, outside the submitted work. D.K., F.S., K.N., K.H., Y.K., and Y.I. have no conflicts of interest. 


\section{Case Reports in Oncology}

\begin{tabular}{l|l}
\hline Case Rep Oncol 2021;14:944-949 \\
\hline DOI: 10.1159/000515508 & $\begin{array}{l}\text { @ 2021 The Author(s). Published by S. Karger AG, Basel } \\
\text { www.karger.com/cro }\end{array}$ \\
\hline
\end{tabular}

Sato et al.: Breast Cancer Treatment in a Patient with Spina Bifida

\section{Funding Sources}

No funding was obtained from the private or public sector for this research.

\section{Author Contributions}

F.S., K.N., and A.S. contributed to the conception and design of the study. All authors contributed to the acquisition and analysis of data. F.S., K.N., and A.S. were major contributors in writing the manuscript. All authors have read and approved the final manuscript.

\section{References}

1 Wiener JS, Suson KD, Castillo J, Routh JC, Tanaka ST, Liu T, et al. Bladder management and continence outcomes in adults with spina bifida: results from the National Spina Bifida Patient Registry, 2009 to 2015. J Urol. 2018; 200(1):187-94.

2 Hoy NY, Metcalfe P. Complications following breast cancer therapy in the adult spina bifida population: a case report. Can Urol Assoc J. 2013;7(11-12):E761-3.

3 Malla HP, Park BJ, Koh JS, Jo DJ. Giant intrathoracic meningocele and breast cancer in a neurofibromatosis type I patient. J Korean Neurosurg Soc. 2016;59(6):650-4.

4 Diéras V, Fumoleau P, Romieu G, Tubiana-Hulin M, Namer M, Mauriac L, et al. Randomized parallel study of doxorubicin plus paclitaxel and doxorubicin plus cyclophosphamide as neoadjuvant treatment of patients with breast cancer. J Clin Oncol. 2004;22(24):4958-65.

5 Bear HD, Anderson S, Brown A, Smith R, Mamounas EP, Fisher B, et al. The effect on tumor response of adding sequential preoperative docetaxel to preoperative doxorubicin and cyclophosphamide: preliminary results from National Surgical Adjuvant Breast and Bowel Project Protocol B-27. J Clin Oncol. 2003;21(22):4165-74.

6 Horner-Johnson W, Dobbertin K, Andresen EM, Iezzoni LI. Breast and cervical cancer screening disparities associated with disability severity. Womens Health Issues. 2014;24(1):e147-53.

7 Altman BM, Bernstein A. Disability and health in the United States, 2001-2005. Hyattsville: National Center for Health Statistics; 2008. 